\title{
Overexpression of SOX9 in mouse embryonic stem cells directs the immediate chondrogenic commitment
}

\author{
Jae-Hwan Kim ${ }^{1,2}$, Hyun-Jin Do ${ }^{1}$, \\ Heung-Mo Yang ${ }^{1}$, Jong-Hyun $\mathrm{Oh}^{2}$, \\ Seong-Jun Choi ${ }^{1,2}$, Dong-Ku Kim ${ }^{1,2}$, \\ Kwang-Yul Cha ${ }^{1,2}$ and Hyung-Min Chung ${ }^{1,2,3}$ \\ ${ }^{1}$ Cell and Gene Therapy Research Institute \\ ${ }^{2}$ Graduate School of Life Science and Biotechnology \\ Pochon CHA University \\ Seoul 135-907, Korea \\ ${ }^{3}$ Corresponding author: Tel, 82-2-3468-3391; \\ Fax, 82-2-3468-3373; E-mail, hmchung@chacares.com
}

Accepted 30 May 2005

Abbreviations: HMG, high mobility group; LIF, leukemia inhibitory factor; mES cell, mouse embryonic stem cell; MSC, mesenchymal stem cell; SOX, sry-type HMG box

\begin{abstract}
Mouse embryonic stem (mES) cells are capable of undergoing chondrogenesis in vitro. To enhance this process, the human SOX9 (hSOX9) cDNA was delivered into mES cells and the clones overexpressing hSOX9 (denoted as mES-hSOX9 cells) were verified by Western blot analysis. The transcripts of collagen IIA (a juvenile form), aggrecan and Pax1 were expressed in mES-hSOX9 cells grown on feeder layers, suggesting the immediate effect of exogenous SOX9 on chondrogenesis. However, SOX9 overexpression did not affect the cell cycle distribution in undifferentiated $\mathrm{mES}$ cells. Upon differentiation, collagen IIB (an adult form) was detected in day 3 immature embryoid bodies. In addition, the overexpression of exogenous SOX9 significantly induced transcriptional activity driven by SOX9 binding site. Taken together, we for the first time demonstrated that constitutive overexpression of exogenous $S O X 9$ in undifferentiated mES cells might have dual potentials to induce both chondrogenic commitment and growth capacity in the undifferentiated status.
\end{abstract}

Keywords: cell differentiation; chondrogenesis; sox9 transcription factor; stem cells; transfection

\section{Introduction}

Articular cartilage is a complex of avascular tissues containing plenty of extracellular matrices including the molecules of collagens II, IX, XI and aggrecan, which functions as a shock absorber and provides a smooth joint movement. Once articular cartilage is damaged by trauma or degenerative arthritis, the complete repair of damaged cartilage is highly problematic mostly due to avascularity and low cellularity (Tsuchiya et al., 2003). Currently, the reconstitution of damaged cartilage is achieved by injection of growth factors attached in biodegradable matrices, tissue transplantation and cellular engraftment (Buckwalter, 2002; Tanaka et al., 2004). Recently, it has been interested in bone marrow-derived mesenchymal stem cells (MSC) as a good candidate for the source of articular cartilage repair because of their potentials to differentiate into various cell types of mesenchymal lineages such as chondrocytes, osteoblasts and adipocytes (Pittenger et al., 1999; Tanaka et al., 2004). However, MSC is a very limited source for tissue engineering due to the impaired growth potentials followed by aging (Tanaka and Liang, 1995; Tanaka et al., 1999).

Mouse embryonic stem (mES) cells, first isolated from the inner cell mass of blastocysts (Martin, 1981), are defined as a stem cell line characterized by infinite proliferation capacities and pluripotentials to differentiate into various cell lineages (Wiles and Keller, 1991; Bain et al., 1995; Tanaka et al., 2004). Because of their characteristics, mES cells have been a good model system to study on in vitro differentiation into various cell types, such as cardiomyocytes, neurons, epithelial cells, and so on (Guan et al., 1999). It has been also reported that mES cells undergo chondrogenic differentiation by cytokine stimulation or various culture conditions (Kramer et al., 2000; Hegert et al., 2002; Tanaka et al., 2004) under a long-term culture system. Moreover, to enhance the efficiency of specified differentiation and obtain the homogenous cell populations, genetic manipulation has been accomplished by introducing exogenous genes into $\mathrm{mES}$ cells such as neurons or hepatocytes (Gratsch et al., 2002; Ishizaka et al., 2002). As the key regulators for chondrogenesis, several transcription factors are expressed in mesenchymal cells during early chondrogenesis, such as high-mobility group (HMG) box protein SOX9, and the paired-box-gene Pax-1 (Wallin et al., 1994; Wright et al., 1995). SOX9, 
as a good candidate to enhance chondrogenic differentiation through genetic modification, is the member of the SOX (Sry-type HMG box) family and predominantly expressed during mesenchymal condensation and cartilage formation in mouse embryos (Wright et al., 1995). In addition, SOX9 is known to be a key regulator for the expression of collagen II and aggrecan, the major matrix proteins specific for chondrocytes through binding to the enhancer region (Bell et al., 1997; Lefebvre et al., 1997; Sekiya et al., 2000). In this study, to enhance the chondrogenic differentiation through genetic modification, we for the first time established the stable cell line overexpressing SOX9 in undifferentiated mES cells and analysed the expression of chondrogenic marker genes.

\section{Materials and Methods}

\section{Cell culture}

Mouse ES (129/Sv, AB2.2) cells on STO feeder cells were cultured in DMEM supplemented with $15 \%$ FBS (Hyclone, Logan, UT), 1 mM $\beta$-mercaptoethanol, 0.1 mM Non-Essential Amino Acid, 100 IU penicillin, 100 $\mu \mathrm{g} / \mathrm{ml}$ streptomycin (Invitrogen, Carlsbad, CA) and $1,000 \mathrm{U} / \mathrm{ml}$ mouse leukemia inhibitory factor (LIF; Chemicon, Temecula, CA). STO and NIH313 cells were cultured in DMEM supplemented with $10 \%$ FBS, $100 \mathrm{IU}$ penicillin and $100 \mu \mathrm{g} / \mathrm{ml}$ streptomycin. For the formation of embryoid bodies for 3 days, STO feeder cells were removed by plating on gelatin-coated tissue culture dish for 30 min twice and then on bacteriological grade petri dish for another $30 \mathrm{~min}$ at $37^{\circ} \mathrm{C}$ incubator.

\section{Plasmid constructs}

A full-length human SOX9 cDNA amplified from human SOX9 clone (ATCC) was inserted into pIRES2EGFP vector (Clontech, Palo Alto, CA) and the hEF1 $\alpha$ promoter region obtained from the pTracer-EF/V5-His $A$ vector (Invitrogen) was replaced with the CMV promoter region of pIRES2-EGFP. Flag epitope tag was added to the $\mathrm{N}$-terminus of the hSOX9 open reading frame. A minimal promoter construct, pGL3ti (Jonk et al., 1998), was prepared from pGL3-basic (Promega, Madison, WI) by inserting the adenovirus major late promoter TATA box and the mouse terminal deoxynucleotidyl transferase gene initiator sequence into the multiple cloning site. SOX9 binding site-driven luciferase reporter construct was prepared by inserting a 48 bp mouse collagen II intron 1 sequence containing sOX9 binding site (CTG TGA ATC GGG CTC TGT ATG CGC TTG AGA AAA GCC CCA TTC ATG AGA; Zhou et al., 1998) into the upstream of the minimal promoter in pGL3ti vector ( $p$ GL3ti-SOX9 BS). All plas- mid constructs were verified by DNA sequencing.

\section{Establishment of stable cell lines in $\mathrm{mES}$ cells}

Mouse ES cells were transfected with linearized pIRES2-hEF1 $\alpha$-EGFP or pIRES2-hEF1 $\alpha$-hSOX9-EGFP expression vector by electroporation with a single pulse of $270 \mathrm{~V} / 500 \mu \mathrm{F}$ and selected with $400 \mu \mathrm{g} / \mathrm{ml}$ G418 treatment (Invitrogen). Each independent clone of transfected mES cells was initially cultured in 48-well plates and propagated in $100 \mathrm{~mm}$ tissue culture dish for further analysis. To obtain neomycinresistant feeder cells, STO cells were transfected with linearized pcDNA 3.0 (Invitrogen) by using FuGene 6 transfection reagents (Roche, Mannheim, Germany)

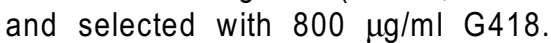

\section{Western blot analysis}

The selected clones of transfected mouse ES cells were cultured in a $100 \mathrm{~mm}$ tissue culture dish and then washed twice with PBS and solubilized in extraction buffer containing $50 \mathrm{mM}$ Tris $(\mathrm{pH} 8.0), 150$ $\mathrm{mM} \mathrm{NaCl}, 5 \mathrm{mM}$ EDTA, 1\% NP-40, $0.1 \%$ SDS, 0.5 $\%$ sodium deoxycholate, $1 \mathrm{mM}$ DTT and $1 \mathrm{X}$ protease inhibitor cocktails. $20 \mu \mathrm{g}$ whole lysates were separated on $8 \%$ SDS-polyacrylamide gels and transferred into polyvinylidene difluoride membrane (Amersham Pharmacia Biotech, Piscataway, NJ). Proteins bound to anti-Flag monoclonal antibody (Sigma, St. Louis, MO) were detected with alkaline phosphataseconjugated secondary antibody (Santa Cruz Biotech, Santa Cruz, CA) and visualized with nitro blue tetrazolium/5-bromo-4-chloro-3-indolyl phosphate (Sigma) substrates.

\section{Cell cycle analysis}

Either empty vector- or hSOX9-transfected mES cells grown on STO feeder cells in complete ES growing medium were rinsed once in chilled PBS, then trypsinized briefly and resuspended in $10 \mathrm{ml}$ ES growing media. Feeder cells were removed by plating the resuspended cells on tissue culture dish for $30 \mathrm{~min}$ twice and then on petri dish for another $30 \mathrm{~min}$ in $37^{\circ} \mathrm{C}$ incubator. The collected cells were pelleted and resuspended in iced-cold PBS. The resuspended cells were fixed by adding $100 \%$ ethanol while vortexing. The cells were kept on $4^{\circ} \mathrm{C}$ for $1 \mathrm{~h}$, pelleted, and then resuspended with PBS containing $50 \mu \mathrm{g} / \mathrm{ml}$ RNase A. Followed by the incubation at room temperature for $5 \mathrm{~min}$, the cells were further incubated in PBS containing $50 \mu \mathrm{g} / \mathrm{ml}$ propidium iodide at $37^{\circ} \mathrm{C}$ incubator for $30 \mathrm{~min}$. Nuclei were analysed in a FACSVantage SE (BD Biosciences, Palo Alto, CA). 


\section{RT-PCR analysis}

Total RNAs were extracted from transfected mouse ES cells by Trizol reagents (Invitrogen) and treated with DNase (Promega). Reverse transcription was carried out using the Superscript II (Invitrogen) and oligo-dT primers (Promega) under the condition of

Table 1. Oligonucleotide primers used for reverse-transcriptionpolymerase chain reaction and expected product sizes.

\begin{tabular}{|c|c|c|}
\hline Targets & Sequences & $\begin{array}{l}\text { Product } \\
\text { sizes (bp) }\end{array}$ \\
\hline \multicolumn{3}{|c|}{ hSOX9 } \\
\hline \multicolumn{3}{|c|}{ F: 5'-CCCGATCTGAAGAAGGAGAGC-3' } \\
\hline & : 5'-GTTCTTCACCGACTTCCTCCG-3' & 381 \\
\hline \multicolumn{3}{|c|}{ mCollagen } \\
\hline \multicolumn{3}{|c|}{$\| \mathrm{A} / \mathrm{B}$} \\
\hline & : 5'-CTGCTCATCGCCGCGGTCCTA -3' & 4321 \\
\hline & : 5'-AGGGGTACCAGGTTCTCCATC -3' & 225 \\
\hline \multicolumn{3}{|c|}{$\mathrm{mPax} 1$} \\
\hline & : 5'-GATGGAAGACTGGGCGGGTGTGAA-3' & 318 \\
\hline \multicolumn{3}{|c|}{ R: 5'-TTCTCGGTGTTTGAAGGTCATTGCCG-3' } \\
\hline \multicolumn{3}{|c|}{ mAggrecan } \\
\hline & : 5'-CCAAGTTCCAGGGTCACTGTTACCG-3' & 270 \\
\hline \multicolumn{3}{|c|}{ R: 5'-TCCTCTCCGGTGGCAAAGAAGTTG-3' } \\
\hline \multicolumn{3}{|c|}{$\mathrm{m} \beta$-Actin } \\
\hline & : 5'-AGGCTGTGCTGTCCCTGTATGC-3' & 373 \\
\hline & : 5-ACCCAAGAAGGAAGGCTGGAAA-3 & \\
\hline
\end{tabular}

A

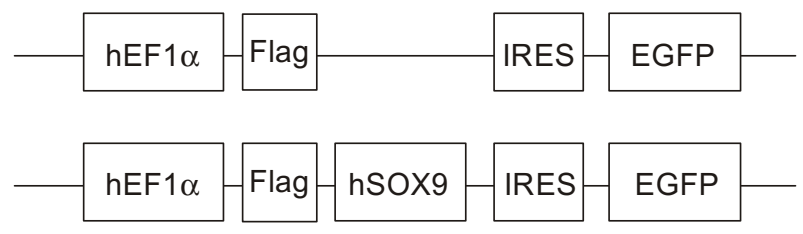

C

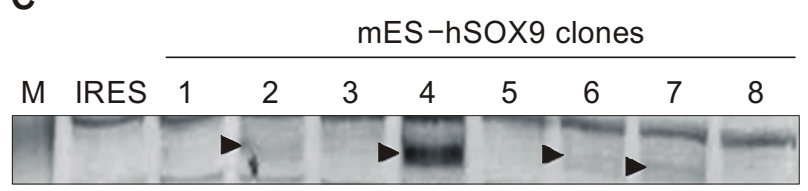

incubation at $42^{\circ} \mathrm{C}$ for $1 \mathrm{hr}$ and $72^{\circ} \mathrm{C}$ for $15 \mathrm{~min}$. The PCR reactions were performed by using DNA Taq polymerase (Promega) with the following conditions: 1 cycle at $94^{\circ} \mathrm{C}$ for $5 \mathrm{~min}$ followed by 35 cycles at $94^{\circ} \mathrm{C}$ for $30 \mathrm{~s}, 60^{\circ} \mathrm{C}$ for $30 \mathrm{~s}, 70^{\circ} \mathrm{C}$ for $1 \mathrm{~min}$ and a final extension at $72^{\circ} \mathrm{C}$ for $15 \mathrm{~min}$. The PCR products were electrophoresed in a $1.2 \%$ agarose gel and visualized by ethidium bromide staining. The primer sequences and product sizes were described in Table 1.

\section{Reporter assays}

NIH3T3 cells were cotransfected with $0.5 \mu \mathrm{g}$ pGL3ti or pGL3ti-SOX9 BS vector and $1 \mu \mathrm{g}$ hSOX9 expression vector by using lipofectamine plus transfection reagent (Invitrogen) in 6-well tissue culture dish. The luciferase activity was normalized to the cotransfected internal control plasmid pcDNA3.1/hygro/LacZ (Invitrogen). Cells were harvested $48 \mathrm{~h}$ after transfection and luciferase activities were measured by using firefly luciferase assay system (Promega). Transfection and reporter assay experiments were carried out in duplicates and independently replicated at least twice.

\section{Results}

Establishment of stable cell lines overexpressing SOX9 in mouse ES cells

It has been reported that the hEF1 $\alpha$ promoter robustly

\section{B}

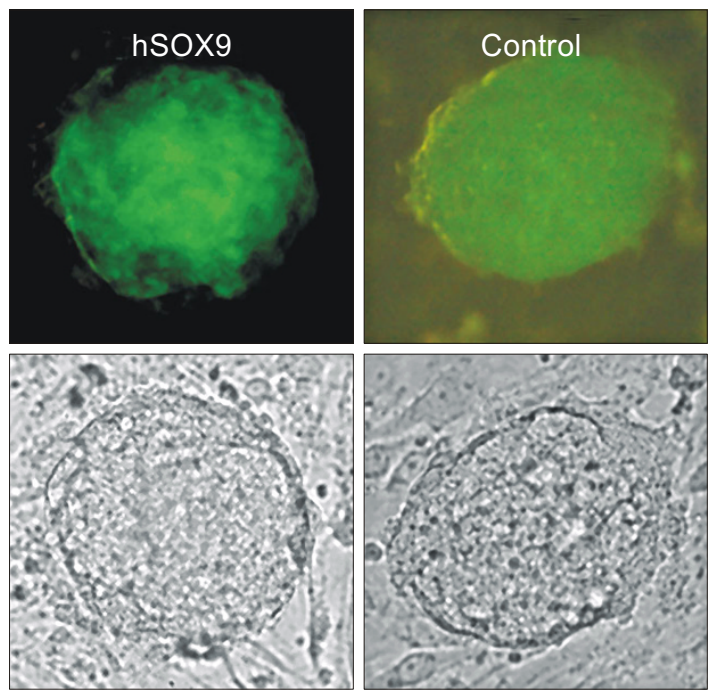

Figure 1. Establishment of stable cell lines overexpressing hSOX9 in mouse ES cells, (A) Vector constructs hSOX9 cDNA was inserted to pIRES2-EGFP and Flag epitope tag was added to the N-terminus of the hSOX9 open reading frame, followed by the modification of promoter system with $h E F 1 \alpha$ (B) Neomycin-resistant clones were selected with G418 treatment and fluorescent microscopic observation of enhanced green fluorescence protein (EGFP) expression; (C) Western blot analysis of mouse ES cells expressing hSOX9 by using anti-Flag antibody. The arrowheads indicate hSOX9 detected with anti-Flag antibody and only 4 out of 8 clones expressed hSOX9 proteins. 
drove the reporter activity while the CMV promoter was inactive in undifferentiated mES cells (Chung et al., 2002). In this study, hSOX9 cDNA was cloned into a bicistronic expression vector pIRES2-EGFP under the control of hEF $1 \alpha$ promoter in order to induce hSOX9 expression effectively and for efficient selection. To enhance chondrogenic differentiation, the cloned hSOX9 expression vector was delivered into $\mathrm{mES}$ cells by electroporation with a single pulse of $270 \mathrm{~V} / 500 \mu \mathrm{F}$. The hSOX9-containing clones were selected with $400 \mu \mathrm{g} / \mathrm{ml} \mathrm{G418} \mathrm{treatment} \mathrm{and} \mathrm{fluore-}$ scence microscopic observation (Figure $1 \mathrm{~A}$ and $1 \mathrm{~B}$ ). Each independent clone was carefully detached by a pasteur glass pippet under a dissect microscopy and propagated for the further analysis. The expression of hSOX9 proteins was confirmed by Western blot analysis with anti-Flag antibody (Figure $1 \mathrm{C}$ ). Despite of dual selection of SOX9-overexpressing clones by

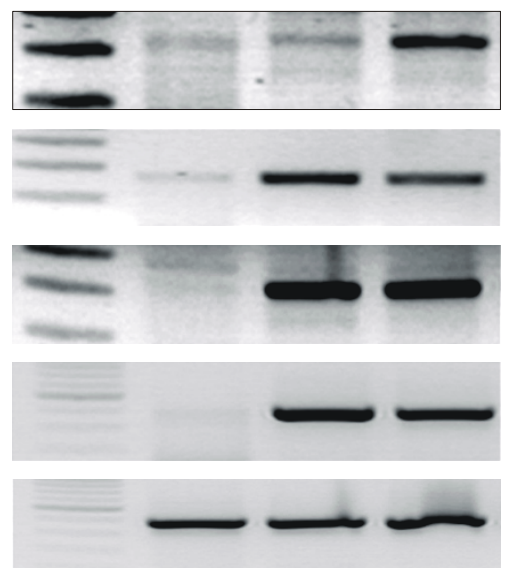

Figure 2. Expression analysis of SOX9 mRNA and chondrogenic marker genes in mES-IRES and mES-hSOX9 cells by RT-PCR.
G418 treatment and fluorescence observation, only 4 out of 8 clones expressed hSOX9 proteins. We chose two clones with differential expression level of hSOX9 for further experiments.

\section{Expression of chondrogenic marker genes in mES-hSOX9 cells}

First of all, we reconfirmed the strong expression of hSOX9 mRNAs in hSOX9-overexpressing mES (designated as mES-hSOX9) cells by RT-PCR analysis (Figure 2). Thereafter, to examine the immediate effect of exogenous SOX9 overexpression on chondrogenesis, we analysed the representative chondrogenic markers by RT-PCR (Figure 2). The transcripts of aggrecan, collagen IIA, and Pax1 were strongly expressed in mES-hSOX9 cells, compared to the control (designated as mES-IRES) cells. However, faint expression of SOX9 transcripts was also observed in mES-IRES due to the cross-reactivity of SOX9 primers between human and mouse.

\section{Exogenous SOX9 does not alter cell cycle pattern in the undifferentiated $\mathrm{mES}$ cells}

We examined whether the delivery of exogenous SOX9 and the expression of chondrogenic marker genes might affect the changes in cell cycle pattern of undifferentiated mES cells. Flow cytometric analysis revealed that the mES-hSOX9 cells did not undergo the changes in cell cycle distribution, compared to mES-IRES cells (Figure 3). However mild increase of G1 ( $3-5 \%$, respectively) was observed in mEShSOX9 clones.

\section{Collagen IIB, an adult form was expressed during embryoid body formation}

When we examined the immediate effect of exogenous SOX9 expression on chondrogenesis, relatively higher expression level of juvenile form, collagen IIA, was observed in mES-hSOX9 cells, compared to
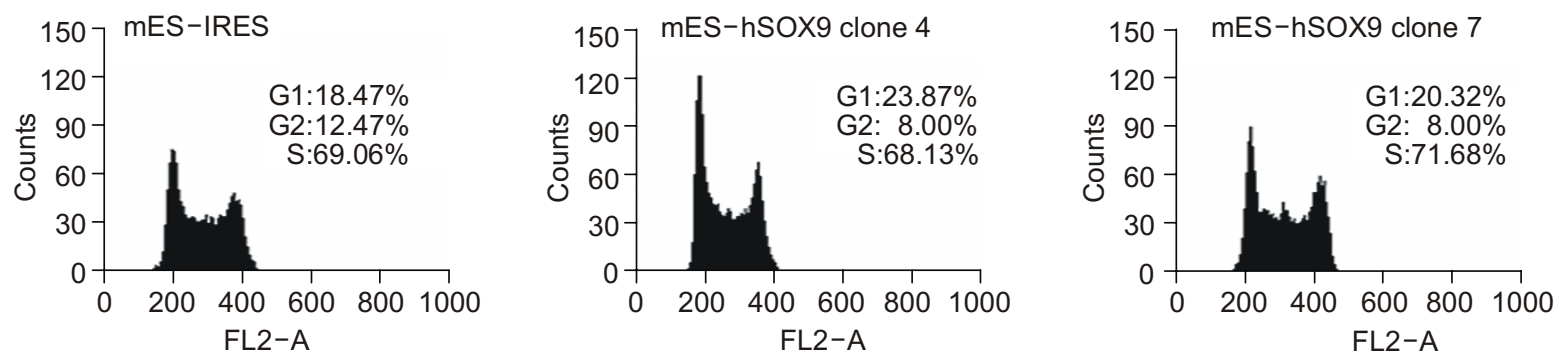

Figure 3. Cell cycle analysis of mES-IRES and mES-hSOX9 cells by flow cytometry. Cells were cultured for 2 days in complete mES growing media and analysed for cell cycle distribution. The percentage of cells in the $G 1, S$ and $G 2$ phases of the cell cycle were calculated from three independent experiments, using the ModFit LT software. 


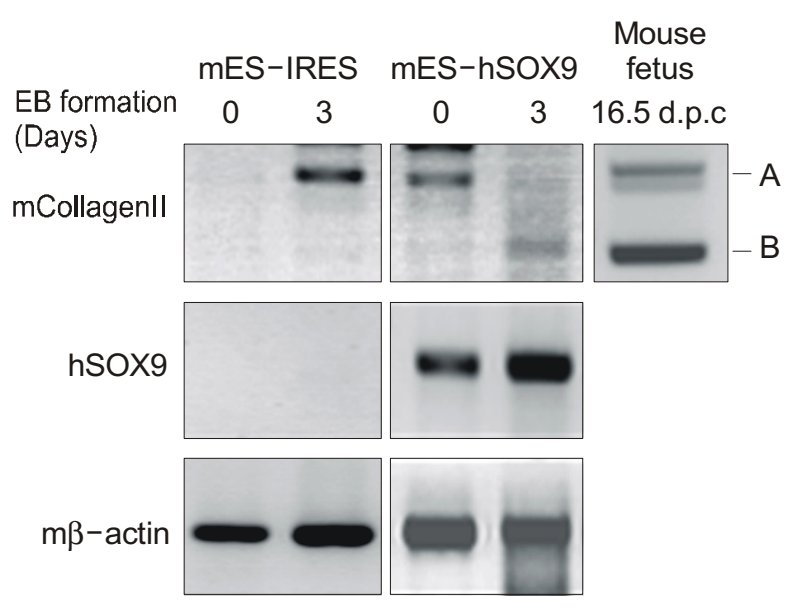

Figure 4. Expression analysis of collagen II in mES-IRES and -hSOX9 cells during embryoid body formation by RT-PCR. The forms of collagen II were indicated with an arrowhead (type $A$, a juvenile form) and an arrow (type B, an adult form).

mES-IRES cells. However, the expression of adult form, collagen IIB was not detected in either of them (Figure 2). To examine whether overexpression of SOX9 could further induce the expression of collagen IIB, an adult form important for functional chondrocyte differentiation, we induced embryoid body formation of the transfected mES cells. RT-PCR analysis revealed that the expression of collagen IIA was significantly decreased and collagen IIB was detected in as early as day 3 embryoid bodies of mES-hSOX9 cells (Figure 4).

\section{Exogenous SOX9 induces the transcriptional activity through binding to SOX9 binding site derived from mouse collagen II enhancer sequences}

To verify whether the expression of collagen II could be induced by overexpression of SOX9, an SOX9 binding site-driven luciferase reporter construct was transiently cotransfected with hSOX9 expression vector in NIH3T3 cells. As a control, the reporter construct without the SOX9 binding site was used. Reporter assay revealed that cotransfection of hSOX9 induced higher transcriptional activity of the reporter containing SOX9 binding site (pGL3ti-SOX9 BS), compared to that of pGL3ti reporter being devoid of SOX9 binding site (Figure 5). This result suggests that overexpression of SOX9 may induce the expression of collagen II by binding to SOX9 binding site within the collagen II gene in mES cells.

\section{Discussion}

In this study, we presented that mES-hSOX9 cells

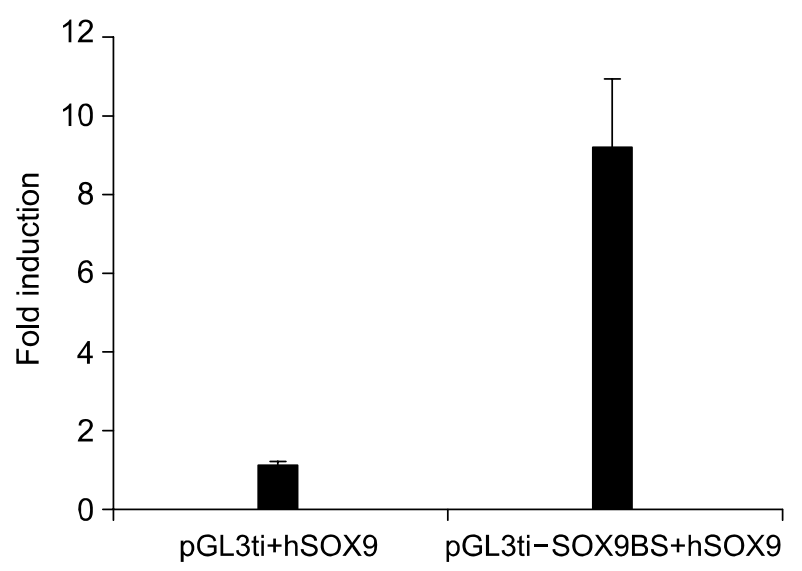

Figure 5. SOX9-mediated stimulation of SOX9 binding site-driven transcription. NIH3T3 cells were cotransfected with $1 \mu \mathrm{g}$ hSOX9 expression vector and $0.5 \mu \mathrm{g}$ reporter plasmid containing 48-bp SOX9 binding site (pGL3ti-SOX9 BS) or no SOX9 binding site (pGL3ti). The data shown are the means and SD of duplicate measurements obtained from one representative transfection. This experiment was performed at least twice in duplicate.

grown on feeder cells could express chondrogenic marker genes such as collagen IIA, aggrecan, and Pax1 without exogenous differentiational stimulation. Moreover, the expression of exogenous SOX9 and chondrogenic markers did not affect the cell cycle pattern of undifferentiated mES cells. However, during differentiation process, collagen IIB, an adult form was detected in as early as day 3 embryoid bodies of mES-hSOX9 cells.

Extensive studies on in vitro chondrogenic differentiations and the molecular mechanisms have been performed in mesenchymal cells (Ahrens et al., 1993; Erlebacher et al., 1995; Mackay et al., 1998; Bai et al., 2004; Ikeda et al., 2004). Recently, it has been reported that even primary mouse embryonic fibroblasts can enter and complete the program of chondrogenic differentiation under the appropriate conditions (Lengner et al., 2004). However, to date, mES cells are obviously good source for cartilage regeneration due to the characteristics of immortality and pluripotentials. mES cells undergo chondrogenic differentiation by cytokine stimulation or different culture conditions (Kramer et al., 2000; Hegert et al., 2002; Tanaka et al., 2004) under a long-term culture system. However, the prolonged differentiation systems would delay the treatment to patients or could alter the immunogenicity of cultured autologous cells, ultimately leading to immunorejection upon transplantation (Hodgetts et al., 2000; Smythe et al., 2000; Heng et al., 2004). To enhance the chondrogenic differentiation through genetic modification, we successfully established stable cell lines constitutively expressing exogenous SOX9 in mES cells. The ex- 
pression of exogenous SOX9 was confirmed by western blot analysis with anti-Flag antibody (Figure 1C).

SOX9 is the member of the SOX (Sry-type HMG box) family and predominantly expressed during mesenchymal condensation and cartilage formation in mouse embryos (Wright et al., 1995). In addition, SOX9 is known to be a key regulator for the expression of collagen II, IX, XI, and aggrecan, the major matrix proteins specific for chondrocytes through binding to the enhancer region (Bell et al., 1997; Lefebvre et al., 1997; Bridgewater et al., 1998; Sekiya et al., 2000; Bernard et al., 2003). SOX9 has been also reported to be required for chondrogenesis by the generation of SOX9-mutant ES cells and chimeric mice ( $\mathrm{Bi}$ et al., 1999). SOX9-mutant cells showed the different expression patterns of cartilage-specific genes upon embryonic development stage, indicating that SOX9 functions as an early marker to activate the genes during cartilage formation. The establishment of mES-hSOX9 cell lines may provide a good source to study on molecular mechanism of chondrogenesis and ultimately to regenerate functional chondrocytes for clinical applications. In this study, the higher expression of cartilage-specific marker genes, collagen IIA and aggrecan was detected in mES-hSOX9, compared with mES-IRES cells (Figure 2). However, the expression of collagen IIA was detected at relatively basal levels, suggesting other cofactors and optimal differentiation culture conditions may be needed to activate the gene sufficiently. In fact, SOX9 interacts with other cofactors, such as L-Sox5 and Sox6 for dimerization and subsequently activates cartilage-specific marker genes more effectively (Bernard et al., 2003; Ikeda et al., 2004).

$\mathrm{mES}$ cells exhibit the very unique distribution of cell cycle: $15 \%$ in $\mathrm{G} 1,75 \%$ in $\mathrm{S}$, and $10 \%$ in $\mathrm{G} 2 / \mathrm{M}$, respectively, supporting their infinite proliferation potentials in vitro without differentiation under LIF signals (Savatier et al., 2002). Our data revealed the similar cell cycle pattern in mES-IRES and -SOX9 cells to the undifferentiated mES cells (Figure 3 ), suggesting the unique cell cycle distribution of undifferentiated mES cells might be not interfered by the integration of exogenous SOX9 gene as well as drug selection process and long-term culture even if the mild increase of $\mathrm{G} 1$ was observed in mES-hSOX9 cells $(3-5 \%$, respectively), compared to mES-IRES cells (Figure 3). Moreover, mES-hSOX9 cells might not only have more directed and specified differentiation potentials into chondrocytes expressing chondrocyte-specific markers, but also still may have the growth capacities despite of genetic modification through exogenous gene delivery. However, further investigation is needed to understand the molecular and signaling mechanisms of genetically modified
mES cells.

It has been reported that the entire nucleotide sequences of mouse collagen II gene were determined by restriction mapping and the two forms of collagen II (A and B) were generated by alternative splicing process (Metsaranta et al., 1991). Collagen IIA is broadly expressed in embryonic or nonchondrogenic tissues as well as prechondrocytes and mesenchymal cells. The expression of collagen IIB, an isoform lacking a 69 amino acid cysteine-rich domain encoded by exon 2 is specifically localized in differentiating and adult chondrocytes, indicating the differentiation into functional chondrocytes $(\mathrm{Ng}$ et al., 1993; Sandell et al., 1994; Valcourt et al., 2003). Moreover, the high expression level of total collagen II and an isoform IIB in a primary culture of chondrocytes dramatically decreased within a few days followed by the first passage, whereas the expression level of collagen IIA relatively increased (Valcout et al., 2003). mES cells has been reported to undergo chondrogenic differentiation and express collagen IIA in as early as day 5 embryoid body. However, the expression of collagen IIB was detected as early as 14 days after replating day 5 embryoid body (Tanaka et al., 2004). Our study for the first time showed that the expression of collagen IIB was detected as early as day 3 since forming the embryoid bodies of mES-hSOX9 cells, suggesting the successful shift from collagen IIA to IIB gene expression might indicate functional chondrocyte differentiation (Figure 4).

Forty eight bp enhancer sequences in the first intron of mouse collagen II have been shown to strongly increase the promoter activity and minimal 18-bp enhancer sequences within the $48 \mathrm{bp}$ were sufficient to direct cartilage-specific expression (Lefebvre et al., 1996). In addition, it has been reported that the collagen II expression was closely related to SOX9 expression and the minimal enhancer sequences directly bound to the HMG domain binding site of SOX9 (Lefebvre et al., 1997). Our study also confirmed that hSOX9 expression vector sufficiently drove the reporter promoter activity containing mouse collagen II 48-bp enhancer sequences (Figure 5), supporting the expression of collagen II is stimulated by exogenous SOX9 expression in mES cells.

In conclusion, we for the first time established the stable cell lines constitutively overexpressing SOX9 and could observe the expression of chondrocyte differentiation marker genes in mES-hSOX9 cells without specified exogenous stimulation such as growth factors or differentiation culture conditions. However, other cofactors and optimal differentiation culture conditions may be needed to induce sufficient amount of collagen II expression and subsequent chondrogenic differentiation. Further investigation is also required to understand the molecular and signaling 
mechanisms regarding of specified differentiation through genetic modification in pluripotent cells.

\section{Acknowledgment}

This work was supported by grant No. 01-PJ1-PG1$01 \mathrm{CH} 11-0003$ from Ministry of Health \& Welfare, Republic of Korea.

\section{References}

Ahrens M, Ankenbauer T, Schroder D, Hollnagel A, Mayer $\mathrm{H}$, Gross $\mathrm{G}$. Exrpession of human bone morphogenic proteins-2 or -4 in murine mesenchymal progenitor C3H10T1/2 cells induces differentiation into distinct mesenchymal cell lineages. DNA Cell Biol 1993;12:871-80

Bai X, Xiao Z, Pan Y, Hu J, Pohl J, Wen J, Li L. Cartilage-derived morphogenetic protein-1 promotes the differentiation of mesenchymal stem cells into chondrocytes. Bioche Biophys Res Comm 2004;325:453-60

Bain G, Kitchens D, Yao M, Huettner JE, Gottlieb DI. Embroynic stem cells express neuronal properties in vitro. Dev Biol 1995;168:342-57

Bell DM, Leung KKH, Wheatley SC, Ng LJ, Zhou S, Ling WL, Sham MH, Koopman P, Tam PPL, Cheah KSE. Sox9 directly regulates the type II collagen gene. Nat Genet 1997;16:174-8

Bernard P, Tang P, Liu S, Dewing P, Harley VR, Vilain E. Dimerization of SOX9 is required for chondrogenesis, but not for sex determination. Hum Mol Genet 2003;12:1755-65

Bi W, Deng JM, Zhang Z, Behringer RR, de Crombrugghe B. Sox9 is required for cartilage formation. Nat Genet 1999; 22:85-9

Bridgewater LC, Lefebvre V, de Crombrugghe B. Chondrocyte-specific enhancer elements in the col11a2 gene resemble the col2a1 tissue-specifc enhancer. J Biol Chem 1998;273:14998-5006

Buckwalter JA. Articular cartilage injuries. Clin Orthop 2002; 402:21-37

Chung S, Andersson T, Sonntag KC, Bjorklund L, Isacson $\mathrm{O}$, Kim KS. Analysis of different promoter systems for efficient transfene expression in mouse embryonic stem cell lines. Stem Cells 2002;20:139-45

Erlebacher A, Filvaroff EH, Gitelman SE, Derynck R. Toward a molecular understanding of skeletal development. Cell 1995;80:371-8

Gratsch TE, O'Shea KS. Noggin and chordin have distinct activities in promoting lineage commitment of mouse embryonic stem (ES) cells. Development 2002;245:83-94

Guan K, Rohwedel J, Wobus AM. Embryonic stem cell differentiation models: cardiogenesis, myogenesis, neurogenesis, epithelial and vascular smooth muscle cell differentiation in vitro. Cytotechnology 1999;30:211-6

Hegert C, Kramer J, Hargus G, Muller J, Guan K, Wobus AM, Muller PK, Rohwedel J. Differentiation plasticity of chondrocytes derived from mouse embryonic stem cells. J Cell Sci 2002;115:4617-28

Heng BC, Cao T, Lee EH. Directing Stem cell differentiation into the chondrogenic lineage in vitro. Stem Cells 2004; 22:1152-67

Hodgetts SI, Beilharz MW, Scalzo AA. Why do cultured transplanted myoblasts die in vivo? DNA quantification shows enhanced survival of donor male myoblasts in host mice depleted of $\mathrm{CD}^{+}$and $\mathrm{CD} 8^{+}$cells or $\mathrm{Nk}^{+} \cdot 1^{+}$cells. Cell Transplant 2000;9:489-502

Ikeda T, Kamekura S, Mabuchi A, Kou I, Seki S, Takato T, Nakamura K, Kawaguchi H, Ikegawa S, Chung UI. The combination of SOX5, SOX6, and SOX9 (the SOX Trio) provides signals sufficient for induction of permanent cartilage. Arthritis Rheum 2004;50:3561-73

Ishizaka S, Shiroi A, Kanda S, Yoshikawa M, Tsujinoue H, Kuriyama S, Hasuma T, Nakatani K, Takahashi K. Development of hepatocytes from ES cells after transfection with the HNF-3 $\beta$ gene. FASEB J 2002;16:1444-6

Jonk LJC, Itoh $\mathrm{S}$, Heldin $\mathrm{CH}$, ten Dijke $\mathrm{P}$, Kruijer W. Identification and functional characterization of a smad binding element (SBE) in the JunB promoter that acts as a transforming growth factor- $\beta$, activin, and bone morphogenetic protein-inducible enhancer. J Biol Chem 1998;273: 21145-52

Kramer J, Hegert C, Guan K, Wobus AM, Muller PK, Rohwedel H. Embryonic stem cell-derived chondrogenic diffrentiation in votro: Activation by BMP-2 and BMP-4. Mech Dev 2000;92:193-205

Lefebvre V, Huang W, Harley VR, Goodfellow PN, de Combrugghe B. Sox9 is a potent activator of the chondrocyte-specific enhancer of the proo1(II) collagen gene. Mol Cell Biol 1997;17:2336-46

Lefebvre V, Zhow G, Mukhopadhyay K, Smith CN, Zhang $Z$, Eberspaecher $H$, Zhou $X$, Sinha $S$, Maity $S N$, de Crombrugghe B. An 18-base-pair sequence in the mouse Proo1(II) collagen gene is sufficient for expression in cartilage and binds nuclear proteins that are selectively expressed in chondrocytes. Mol Cell Biol 1996;16:4512-23

Lengner CJ, Lepper C, Van Wijen AJ, Stein JL, Stein GS, Lian JB. Primary mouse embryonic fibroblasts: a model of mesenchymal cartilage formation. J Cell Physiol 2004;200: 327-33

Mackay AM, Beck SC, Murphy JM, Barry FP, Chichester CO, Pittenger MF. Chondrogenic differentiation of cultured human mesenchymal stem cells from marrow. Tissue Eng 1998;4: 415-28

Martin GR. Isolation of a pluripotent cell line from early mouse embryos cultured in medium conditioned by teratocarcinoma stem cells. Proc Natl Acad Sci USA 1981;78: 7634-8

Metsaranta $M$, Toman $D$, de Crombruggle B, Vuorio E. Mouse type II collagen gene. J Biol Chem 1991;266:16862-9

$\mathrm{Ng}$ LJ, Tam PP, Cheah KS. Preferential expression of alternatively spliced mRNAs encoding type II procollagen with a cysteine-rich amino-propeptide in differentiating cartilage and nonchondrogenic tissues during early mouse 
development. Dev Biol 1993;159:403-17

Pittenger MF, Mackay AM, Beck SC, Jaiswal RK, Douglas R, Mosca JD, Moorman MA, Simonetti DW, Craig S, Marshak DR. Multilineage potential of adult human mesenchymal stem cells. Science 1999;284:143-7

Sandell LJ, Nalin AM, Reife RA. Alternative splice form of type II procollagen mRNA (IIA) is predominant in skeletal precursors and non-cartilaginous tissues during early mouse development. Dev Dyn 1994;199:129-40

Savatier P, Lapillonne H, Jirmanova L, Vitelli L, Samarut J. Analysis of the cell cycle in mouse embryonic stem cells. Methods Mol Biol 2002;185:27-33

Sekiya I, Tsuji K, Koopman P, Watanabe H, Yamada $\mathrm{Y}$, Shinomiya K, Nifuji A, Noda M. Sox9 enhances aggrecan gene promoter/enhancer activity and is up-regulated by retinoic acid in a cartilage-derived cell, TC6. J Biol Chem 2000;275:10738-44

Smythe GM, Grounds MD. Exposure to tissue culture conditions can adversely affect myoblast behavior in vivo in whole muscle grafts: implications for myoblast transfer therapy. Cell Transplant 2000;9:379-93

Tanaka $\mathrm{H}$, Liang $\mathrm{CT}$. Effect of platelet-derived growth factor on DNA synthesis and gene expression in bone marrow stromal cells derived from adult and old rats. J cell Physiol 1995;164:367-75

Tanaka H, Murphy CL, Murphy C, Kimura M, Kawai S, Polak JM. Chondrogenic differentiation of murine embryonic stem cells: Effects of culture conditions and Dexamethasone. J
Cell Biochem 2004;93:454-62

Tanaka $\mathrm{H}$, Ogasa $\mathrm{H}$, Barnes J, Liang CT. Actions of bFGF on mitogenic activity and lineage expression in rat osteoprogenitor cells: Effect of age. Mol Cell Endocrinol 1999; 150:1-10

Tsuchiya $H$, Kitoh $H$, Sugiura $F$, Ishiguro $N$. Chondrogenesis enhanced by overexpression of sox 9 gene in mouse bone marrow-derived mesenchymal stem cells. Biochem Biophys Res Comm 2003;301:338-43

Wallin J, Wilting J, Koseki $\mathrm{H}$, Fritsch R, Christ B, Balling $\mathrm{R}$. The role of Pax-1 in axial skeleton development. Development 1994;120:1109-21

Valcourt U, Gouttenoire J, Aubert-Foucher E, Herbage D, Mallein-Gerin F. Alternative splicing of type II procollagen pre-mRNA in chondrocytes in oppositely regulated by BMP-2 and TGF- $\beta 1$. FEBS Letters 2003;545:115-9

Wiles MV, Keller G. Multiple Hematopoietic lineage develop from embryonic stem (ES) cells in culture. Development 1991;111:259-67

Wright E, Hargrave MR, Christiansen J, Cooper L, Kun J, Evans T, Gangadharan U, Greenfield A, Koopman P. The Sry-related gene Sox9 is expressed during chondrogenesis in mouse embryos. Nat Genet 1995;9:15-20

Zhou G, Lefebvre V, Zhang Z, Eberspaecher H, de Crombrugghe $B$. Three high mobility group-like sequences within a 48-base pair enhancer of the Col2al gene are required for cartilage-specific expression in vivo. J Biol Chem 1998; 273:14989-97 